PREPARED FOR THE U.S. DEPARTMENT OF ENERGY, UNDER CONTRACT DE-AC02-76CH03073

PPPL-3907

PPPL-3907

UC-70

Kinetic Effects on the Stability Properties of Field-reversed Configurations: II. Nonlinear Evolution

by

Elena V. Belova, Ronald C. Davidson, Hantao Ji, and Masaaki Yamada

November 2003
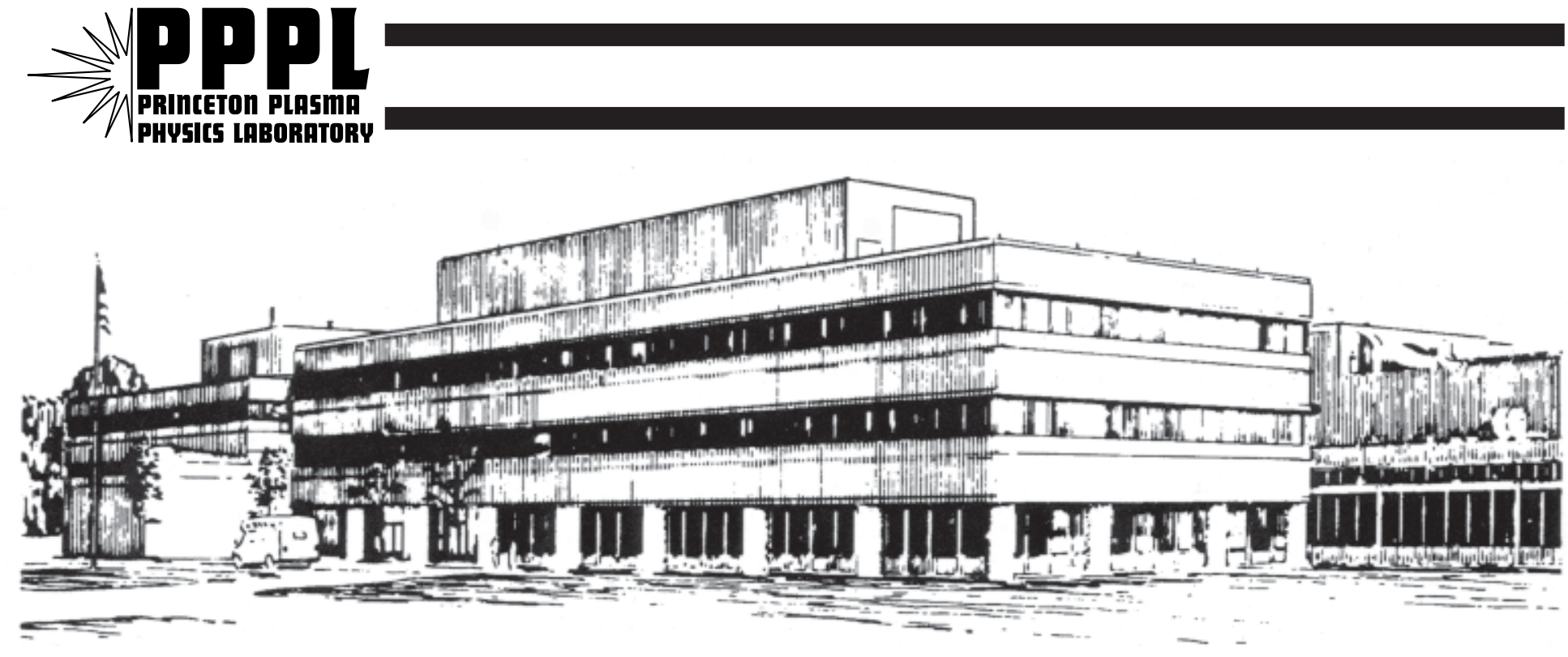

PRINCETON PLASMA PHYSICS LABORATORY PRINCETON UNIVERSITY, PRINCETON, NEW JERSEY 


\section{PPPL Reports Disclaimer}

This report was prepared as an account of work sponsored by an agency of the United States Government. Neither the United States Government nor any agency thereof, nor any of their employees, makes any warranty, express or implied, or assumes any legal liability or responsibility for the accuracy, completeness, or usefulness of any information, apparatus, product, or process disclosed, or represents that its use would not infringe privately owned rights. Reference herein to any specific commercial product, process, or service by trade name, trademark, manufacturer, or otherwise, does not necessarily constitute or imply its endorsement, recommendation, or favoring by the United States Government or any agency thereof. The views and opinions of authors expressed herein do not necessarily state or reflect those of the United States Government or any agency thereof.

\section{Availability}

This report is posted on the U.S. Department of Energy's Princeton Plasma Physics Laboratory Publications and Reports web site in Fiscal Year 2004. The home page for PPPL Reports and Publications is: http://www.pppl.gov/pub_report/

DOE and DOE Contractors can obtain copies of this report from:

U.S. Department of Energy

Office of Scientific and Technical Information

DOE Technical Information Services (DTIS)

P.O. Box 62

Oak Ridge, TN 37831

Telephone: (865) 576-8401

Fax: (865) 576-5728

Email: reports@adonis.osti.gov

This report is available to the general public from:

National Technical Information Service

U.S. Department of Commerce

5285 Port Royal Road

Springfield, VA 22161

Telephone: $1-800-553-6847$ or

(703) $605-6000$

Fax: (703) 321-8547

Internet: http://www.ntis.gov/ordering.htm 


\title{
Kinetic effects on the stability properties of field-reversed configurations: II. Nonlinear evolution
}

\author{
Elena V. Belova, Ronald C. Davidson, Hantao Ji, Masaaki Yamada \\ Plasma Physics Laboratory, Princeton University, Princeton, NJ 08543
}

\begin{abstract}
Results of three-dimensional hybrid simulations of the field-reversed configuration (FRC) are presented. Emphasis of this work is on the nonlinear evolution of magnetohydrodynamic (MHD) instabilities in kinetic FRCs. A wide range of $\bar{s}$ values is considered, where the $\bar{s}$ is the FRC kinetic parameter, which measures the number of ion gyroradii in the configuration. The linear and nonlinear stability of MHD modes with toroidal mode numbers $n \geq 1$ is investigated, including the effects of ion rotation, finite electron pressure, and weak toroidal field. Low- $\bar{s}$ simulations show nonlinear saturation of the $n=1$ tilt mode. The $n \geq 2$ rotational modes are observed to grow during the nonlinear phase of the tilt instability due to ion spin-up in the toroidal direction. Large- $\bar{s}$ simulations show no saturation of the tilt mode, and there is a slow nonlinear evolution of the instability after the initial fast linear growth. Overall, the hybrid simulations demonstrate the importance of nonlinear effects, which are responsible for the saturation of instabilities in low $\bar{s}$ configurations, and also for the increase in FRC life-time compared to MHD models in high- $\bar{s}$ configurations.
\end{abstract}




\section{INTRODUCTION}

Stability properties of the field-reversed configuration (FRC) have remained a mystery for many years, because the predictions of magnetohydrodynamic (MHD) theory and nonlinear MHD simulations have been in contradiction with experimental observations. Experimental FRCs were observed to suffer the $n=2$ rotational instability, which was eventually suppressed by the application of weak multipole magnetic fields after FRC formation ${ }^{1,2}$. The $n=2$ rotational mode is the only experimentally observed global mode, which often prematurely terminates the $\mathrm{FRC}^{1,3}$. The $n=1$ tilt mode, which has been theoretically shown to be strongly unstable within an MHD model, and which is considered to be the most dangerous global mode in the prolate FRC, has not been observed, or it has been seen to "decay away" (in low- $\bar{s}$ cases) without destroying the configuration $^{3-5}$. Significant efforts has been made to include various kinetic effects in theoretical models in order to explain the observed FRC stability properties ${ }^{6-15}$. Linear kinetic calculations have shown a reduction in instability growth rate for small $\bar{s}$ due to finite Larmor radius (FLR) stabilization. However, the growth rate reduction was not large enough to explain the experimental results $^{13-15}$. Other effects have been suggested to further reduce the linear growth rates, in particular the Hall effect, equilibrium toroidal fields, sheared flows, and kinetic electron effects ${ }^{9-11}$. Although more complete models could still provide a stronger reduction in growth rate, the FRC stability issue is far from resolved.

Initial nonlinear simulations, on the other hand, discovered that the $n=1$ tilt instability can saturate nonlinearly in the low $-\bar{s}$ regime ${ }^{16}$. These results suggest that FRC stability properties can be explained provided a combination of kinetic and nonlinear effects is considered. A companion paper ${ }^{13}$ concentrated on linear stability properties, including kinetic and profile effects. Here, the nonlinear evolution of the $n=1$ tilt mode and higher- $n$ modes in prolate FRCs is investigated 
using a hybrid and MHD simulation code $\mathrm{HYM}^{13,14}$. Nonlinear simulations for small $\bar{s}$ values $(\bar{s} \lesssim 2)$ and relatively large $\bar{s}$ values $(\bar{s} \sim 7)$ are performed, and the effects of ion spin-up and particle loss along the open field lines are studied. The nonlinear saturation of the $n=1$ tilt mode, and the growth of the $n \geq 2$ rotational modes are discussed. In addition, the effects of finite electron pressure and weak toroidal field on FRC stability properties are studied, as well as FLR stabilization of the $n>1$ MHD modes in a non-rotating FRC.

The FRC parameters used throughout this paper are defined as follows: the separatrix elongation is the ratio of the separatrix half-length to its radius, $E=Z_{s} / R_{s}$; the kinetic parameter, $S^{*}=R_{s} / \lambda_{i}$, is the ratio of the separatrix radius to the ion skin depth, based on the maximum density, $n_{0}$; another useful kinetic parameter $\bar{s}$ is defined by $\bar{s}=\int_{R_{0}}^{R_{s}} r /\left(R_{s} \rho_{i}\right) d r$, where $R_{0}$ is the magnetic null radius, and $\rho_{i}$ is the local ion Larmor radius. The parameter $\bar{s}$ is approximately equal to the number of ion Larmor radii between the magnetic null and the separatrix. The two kinetic parameters, $S^{*}$ and $\bar{s}$ correspond to different physical stabilizing mechanisms, namely, the Hall term and FLR stabilization, respectively. However, their values are related through the pressure balance condition in cases where the electron pressure can be neglected. The characteristic Alfvén velocity $V_{A}$ is computed using the external field, $B_{\text {ext }}$, and the maximum density; and the Alfvén time is defined as $t_{A}=R_{c} / V_{A}$, where $R_{c}$ is the radius of the flux conserving shell.

\section{NUMERICAL MODEL}

The numerical scheme implemented in HYM code has been described elsewhere ${ }^{14}$. The results presented in the present paper have been obtained using the hybrid version of the HYM code, in which the electrons are described as a fluid, and a fully kinetic (particle) description is used for the ions. The basic equations are 


$$
\begin{aligned}
\frac{\partial \mathbf{B}}{\partial t} & =-c \nabla \times \mathbf{E}, \\
\mathbf{E} & =-\mathbf{v}_{e} \times \mathbf{B} / c-\nabla p_{e} / e n_{e}+\eta \mathbf{J}, \\
\mathbf{J} & =c / 4 \pi \nabla \times \mathbf{B}, \\
\mathbf{v}_{e} & =-\left(\mathbf{J}-\mathbf{J}_{i}\right) / e n_{e}, \\
\frac{\partial p_{e}}{\partial t} & +\gamma p_{e}\left(\nabla \cdot \mathbf{v}_{e}\right)+\mathbf{v}_{e} \cdot \nabla p_{e}=\eta(\gamma-1) J^{2},
\end{aligned}
$$

where electron inertia effects and the displacement current are neglected, and quasineutrality $n_{e}=$ $n_{i}$ is assumed. The ion density and current density are calculated from the distribution of the simulation particles, which follow the ions trajectories calculated using the Lorenz-force equations

$$
\begin{aligned}
& \frac{d \mathbf{x}}{d t}=\mathbf{v}, \\
& \frac{d \mathbf{v}}{d t}=\frac{q_{i}}{m_{i}}(\mathbf{E}-\eta \mathbf{J}+\mathbf{v} \times \mathbf{B} / c) .
\end{aligned}
$$

The delta-f particle simulation method ${ }^{17}$ is employed in order to reduce the numerical noise level. In the delta-f method, the equilibrium ion distribution function $f_{0}$ is assumed to be known analytically, and the perturbed distribution function $\delta f=f-f_{0}$ is integrated along the particle trajectories in Eqs.(6) and (7). Each simulation particle is assigned a weight $w=\delta f / g$, which is evolved in time according to

$$
\frac{d w}{d t}=-\left(\frac{f}{g}-w\right) \frac{d}{d t} \ln f_{0}
$$

Here $g$ is distribution function of simulation (marker) particles, which can be chosen to be the same as $f$, but in general $g \neq f$. Equation (8) follows from the conservation of $f$ and $g$ along the particle trajectories. The numerical noise is reduced in this scheme, because the equilibrium part of moments of the distribution function is calculated analytically using the known $f_{0}$, and particle weights are used to calculate only the perturbed values according to 


$$
\begin{aligned}
& n_{i}=n_{0}+\delta n_{i}=n_{0}+\sum_{m} w_{m} S\left(\mathbf{x}-\mathbf{x}_{m}\right) \\
& \mathbf{J}_{i}=\mathbf{J}_{i 0}+\delta \mathbf{J}_{i}=\mathbf{J}_{i 0}+\sum_{m} w_{m} \mathbf{v}_{m} S\left(\mathbf{x}-\mathbf{x}_{m}\right),
\end{aligned}
$$

where the sum is over simulation particle indices, and $S(\mathbf{x})$ is a shape function (linear).

The delta-f method, as given by Eqs. (9) and (10), provides a reduction of numerical noise by a factor of $|\delta f / f|^{2}$ compared to conventional particle-in-cell (PIC) simulations. Therefore, this method, although fully nonlinear, is most effective when the perturbation amplitude is relatively small, so that $|w| \ll 1$. Modification of this scheme is needed if the perturbations are allowed to grow to large amplitudes with $\delta f \sim O(1)$, in order to assure accuracy of the numerical results. A modified scheme has been developed, which allows us to switch dynamically from the delta-f method to the regular (i.e. full-f) PIC method, as the perturbation amplitude becomes large. A detailed description of the modified numerical scheme is given in Appendix A.

In this paper, equilibria with zero initial ion rotation have been considered. The equilibrium ion distribution function is taken to be $f_{0}=A \exp \left(-\varepsilon / T_{0}\right)$, where $\varepsilon=m_{i} v^{2} / 2+q_{i} \varphi_{0}$ is the particle energy, $\varphi_{0}$ is the equilibrium electrostatic potential, and $T_{0}$ is the uniform ion temperature. The equilibrium current is assumed to be carried by the electrons, and the force balance condition is $q_{i} n_{i} \mathbf{E}_{0}=\nabla p_{i}$. The nonlinear simulations have been performed with a cylindrical grid size of $50 \times 32 \times 60$ in $(r, \phi, z)$ using $4 \cdot 10^{6}$ simulation particles. Perfectly conducting boundary conditions are applied at the radial boundaries, and periodic boundary conditions are used in the axial direction. The region outside the separatrix (open field lines) is modeled as a low-density, high-resistivity plasma.

\section{STABILITY PROPERTIES OF $n=1-3$ MHD MODES}

Most previous linear studies have concentrated on the $n=1$ tilt mode stability properties, and 
considered simple FRC models which neglect, in particular, the effects of the electron pressure and toroidal fields. In this section, these effects are included in the tilt mode stability calculations, and the kinetic stabilization of the higher $n$ modes is investigated.

In the previous paper $^{13}$, it was demonstrated that ion FLR effects provide a major stabilization mechanism for the $n=1$ tilt mode in prolate FRCs. A significant reduction of the growth rate for long, elliptical configurations was found, with the approximate scaling

$$
\gamma=C \frac{V_{A}}{R_{s} E} \exp \left(-3 E \frac{\rho_{i}}{R_{s}}\right)
$$

which has been obtained from a fit to numerical results for FRC configurations with $\mathrm{E}=4,6.25$ and 11.6. Here, $\gamma_{m h d}=C V_{A} / R_{s} E$ is the growth rate of the tilt mode in the MHD model, $\rho_{i}$ is ion thermal Larmor radius in the external field, and $C \approx 2$ is a constant. The exponential factor in Eq. (11) is due to FLR effects, and the reduction of the linear growth rate depends on the product $E \rho_{i} \sim E / \bar{s}$. Therefore, for a given value of $\bar{s}$, the growth rate reduction is stronger for more elongated configurations. The empirical scaling in Eq. (11) is violated in highly kinetic cases with $E \rho_{i} / R_{s} \gtrsim 0.5-1$, when resonant particle effects become important ${ }^{13}$.

The significance of the ion FLR effects is also seen in linear hybrid simulations with finite electron pressure or finite equilibrium toroidal field. In both cases the growth rate of the tilt mode increases due to the effective reduction of the ion FLR stabilization. Figure 1 (thick line) shows the normalized growth rates of the tilt instability calculated for a fixed-size elliptical configuration with $E=6.25$ and $S^{*}=10$, in which the fraction of the total pressure carried by the electrons has been varied between $P_{e}=0$ and $P_{e}=0.875 P$ (for a given total pressure, this corresponds to a reduction of the ion temperature by a factor of 8). For comparison, the dependence of growth rate $\gamma$ on the $1 / \bar{s}$ parameter is plotted for FRCs with $P_{e}=0$ (thin line), and a dashed line shows the empirical 
FLR scaling in Eq. (11). It can be seen that the reduction of the ion thermal Larmor radius in the finite $P_{e}$ cases accounts for an increase in the tilt mode growth rate. Larger electron pressure can also significantly modify the real frequency of the mode. However for most experimental conditions $P_{e} \lesssim 0.5 P_{i}$, and this effect can be neglected.

A weak toroidal magnetic field with magnitude 0.1-0.3 times the external field has also been found to increase the tilt instability growth rates. Such fields can be present in experimental FRCs, and have been considered as a possible stabilizing factor. However, our simulations with a symmetric (spheromak-like) $B_{\phi}$ profile, elongation $E=6$, and $S^{*}=20$ show an increase of the instability growth rate. For example, for $B_{\phi}=0.2 B_{\text {ext }}$ (at the magnetic axis) the growth rate is increased by a factor of 1.4. This destabilization is related to the reduction of the volume-averaged ion Larmor radius, which appears to be the most significant consequence of adding a weak toroidal magnetic field in the FRC. Note that the value of plasma beta at the magnetic axis in this case is still very large $\beta \sim 25$, which implies that the plasma dynamics cannot be strongly modified by the toroidal field.

Stability properties of the $n=2$ and $n=3$ modes have been studied for FRC equilibria with $E=6.25$ and elliptical separatrix shape. A set of linearized hybrid simulations have been performed for each toroidal mode number and various values of the kinetic parameter $\bar{s}$. Unlike previous $n=2$ rotational instability $\operatorname{studies}^{18}$, zero equilibrium ion rotation has been assumed. Results of the linear stability study are summarized in Fig. 2a, where the normalized growth rates are plotted as a function of $1 / \bar{s}$, and $\gamma_{0}=V_{A} / Z_{s}$. For these equilibria, the most unstable $n=2$ and $n=3$ modes are the co-interchange modes with axial polarization. In the ideal MHD limit, these modes are more unstable than the $n=1$ tilt mode. However, FLR effects strongly reduce the growth rates of the $n \geq 2$ modes, which are stabilized at $\bar{s}=1.2(n=2$ mode $)$ and $\bar{s}=2.4$ 
( $n=3$ mode), respectively. Figure $2 \mathrm{a}$ shows that in the kinetic FRC, the $n=1$ tilt mode is the most unstable mode even for intermediate values of $\bar{s}$, i.e., for $\bar{s} \lesssim 10\left(S^{*} \lesssim 80\right)$.

Finite Larmor radius effects change the structure of the most unstable modes with $n=2$ and 3 compared with the MHD model. In the MHD model, these modes are axially polarized kink-like modes, which have maximum amplitude near the magnetic null. In the kinetic FRC model, the mode amplitude is reduced in the vicinity of the magnetic null, where FLR stabilization is the strongest. The example of the linear structure of the $n=2$ mode for $\bar{s}=4.7$ is shown in Figs. $2 \mathrm{~b}$ and 2c. The unstable $n=3$ mode has a very similar structure, except the $n=3$ mode has a smaller radial extent.

Finite Larmor radius effects on MHD modes other than the $n=1$ mode have been investigated by Iwasawa et al. ${ }^{15}$ using a linear gyroviscous model for relatively large azimuthal mode numbers $n=10$ and 30. In general agreement with our hybrid simulation results, they find that FLR stabilization of the higher- $n$ modes is significantly more effective than that of the $n=1$ tilt mode. However, one should note that the FLR-fluid approach relies on an expansion with $k_{\perp} \rho_{i}<1$, which is not valid for $n \geq 10$ modes in the kinetic regime when $S^{*} \lesssim 30(\bar{s} \lesssim 4)$.

\section{NONLINEAR SIMULATIONS AT LOW $\bar{s}$}

\section{A. Ion spin-up}

Nonlinear hybrid simulations have been performed for two sets of equilibrium configurations with $E=4$ and $E=6.25$, elliptical separatrix shapes, and a range of kinetic parameter values: $\bar{s}=1.2-2.4$. In the initial configuration, all of the equilibrium current is carried by the electrons, and the ions have a nonrotating Maxwellian distribution, which is consistent with experimental conditions just after FRC formation. However, as the simulation proceeds, the ions gradually 
begin to rotate toroidally, and near the end of the simulation run $\left(t \sim 60-80 t_{A}\right)$ the ion spin-up is of order $\alpha=V_{\phi, i} / V_{*} \sim 0.5-1$, where $V_{*}=J_{0} / e n_{0}$ is value of relative drift velocity between the ions and electrons in the initial equilibrium. (For our parameters, the corresponding drift frequency is $\Omega_{*}=0.015-0.06 \omega_{c i}$.)

It is remarkable that similar ion spin-up is observed in theta-pinch-formed FRC experiments ${ }^{1}$; however, the origin of the rotation may be different. Namely, the two major spin-up mechanisms proposed to explain the experimentally observed ion rotation, that is, particle loss and end-shorting of the electric field on open field lines, are absent in our numerical model which assumes periodic axial boundary conditions. Numerically observed ion spin-up, at least partially, can be accounted for by the interaction with the radial wall (due to the finite plasma density $\sim 0.1 n_{0}$ at the wall), and the internal flux loss. It is possible that other effects, particularly the plasma poloidal dynamics, contribute to the spin-up.

In the simulations, the ion rotation is localized initially near the separatrix, but gradually the bulk of the ions inside the separatrix spins-up. At later time, the rigid-rotor-like rotation profile forms. The midplane radial profiles and a contour plot of the ion toroidal velocity obtained in simulations with $E=6.25$ and $\bar{s}=2.4$ at two different times $t=46 t_{A}$ and $t=80 t_{A}$ are shown in Fig. 3. The ions inside the separatrix rotate in the positive (diamagnetic) direction, whereas the ions on open field lines near the FRC ends rotate in the opposite direction. The maximum rotation velocity $(n=0)$ is $V_{\phi, i} \sim 0.1 V_{A}$ for the $\bar{s}=2.4$ configuration. In a more kinetic FRC, with $\bar{s}=1.2$, the ion rotation is about twice as fast, with $V_{\phi, i} \sim 0.2 V_{A}\left(\right.$ at $\left.t \sim 60 t_{A}\right)$.

Aside from the details of the spin-up mechanism, the ion rotation in the nonlinear simulations appears to describe well the experimentally observed behavior. An important consequence of the ion spin-up is its effect on the evolution of the low- $n$ modes in the nonlinear phase of the tilt 
instability.

\section{B. Nonlinear evolution of the $n=1$ tilt mode}

In agreement with the linear results in Section III, the initial phase of the simulations shows slow (compared to the MHD) growth of the $n=1$ tilt mode, which remains a dominant mode until the nonlinear phase. The dominant growth of the tilt mode has been observed in all simulation runs performed for two sets of equilibria (with $E=6.25$ and $E=4$ ), and with different $\bar{s}$ values $(1.2,1.6$, and 2.4), including the simulation runs with random initial conditions.

Figure 4 shows the energy evolution of the first five toroidal modes $n=0-4$ for simulations with $E=6.25$ and $\bar{s}=2.4$. The kinetic energy is normalized to the total magnetic energy. The growth of the $n=0$ mode in Fig. 4 corresponds to the ion toroidal spin-up. The $n=1$ tilt instability grows linearly until $t \approx 40 t_{A}$, when its growth rate reduces substantially. The nonlinear slow-down of the instability may be a result of the change of the ion distribution function and a reduction of the instability drive ${ }^{13}$. The ion sheared rotation, which develops by that time, can also contribute to the stabilization. An estimate of the ion rotation frequency $\Omega_{i} \sim(0.5-1) V_{A} / Z_{s}$ at $t \gtrsim 60 t_{A}$ shows that it is of the same order as the linear growth rate of the tilt mode: $\Omega_{i} \sim \gamma$. Figure 4 shows that the tilt mode amplitude reaches its maximum value of $\left|V_{i}\right|^{2} \sim 4 \cdot 10^{-4}$ at $t \approx 60 t_{A}$, and decreases subsequently. The $n=2$ mode amplitude continues to grow after the saturation of the tilt mode, but it also appears to saturate at $t \approx 75 t_{A}$. The amplitudes of the higher- $n$ modes remain significantly smaller than those of the $n=1$ and $n=2$ modes throughout the simulation. Numerical accuracy can be assessed from the conservation of total energy, which shows that the variation in the total energy is less than 0.1 of the change in the magnetic energy until $t=50 t_{A}$, at which point the simulation is changed from the $\delta f$ method to the conventional PIC scheme. 
Figure 5 shows time evolution of the plasma density for the same simulations as in Fig. 4. To take into account the mode rotation, the poloidal plane, which corresponds to the maximum of the tilt amplitude, is shown at each time. The initial MHD-like tilting, which is seen at $t=40 t_{A}$, is modified later at $t=60-64 t_{A}$ before the tilting motion saturates. The resulting configuration has a flatter density profile, and a larger separatrix beta compared to the initial configuration. The plasma profile at $t \gtrsim 70 t_{A}$ is not completely axisymmetric, but rather shows an elliptical deformation which corresponds to the $n=2$ mode. The vector plot of poloidal magnetic field at $t=80 t_{A}$ is shown in Fig. 6. The degree of field reversal is reduced in accordance with the increased separatrix pressure. No self-generated toroidal $(n=0)$ field has been observed, and the magnitude of the toroidal component of the magnetic field is found to be small.

The nonlinear evolution of the $n=1$ mode in the simulations with different elongations and $\bar{s}$ values has been found to be similar to that shown in Fig. 4. However, the simulation with smaller $\bar{s}(\bar{s}=1.2)$ shows a saturation amplitude of the tilt mode about four times smaller than that in Fig. 4, perhaps due to smaller linear growth rate. In addition, the nonlinear behavior of other MHD modes with $n \geq 2$ is found to vary significantly in these simulations as discussed below.

\section{Nonlinear evolution of $n \geq 2$ MHD modes}

The $n=2$ distortions of the FRC density profile are often observed experimentally and are usually attributed to the $n=2$ rotational instability ${ }^{2,3}$. Such perturbations are also seen in our nonlinear hybrid simulations after saturation of the $n=1$ tilt mode instability (Fig. 7). The growth of the higher- $n$ modes is very likely be related to the ion toroidal spin-up and other nonlinear effects, because, as has been shown linerly in Section III, the $n>1$ modes are less unstable than the $n=1$ tilt mode for all considered FRC equilibria. Thus, for the simulations shown in Figs. 4 and 7, the linear growth rate of the $n=2$ mode is about 2.5 times smaller than that of the tilt mode, 
and the $n=3$ mode is marginally unstable [Fig. 3(a)]. However, the $n=2$ mode becomes the dominant mode in the nonlinear phase of the simulations, and the analysis of the mode structure shows that the growing mode has mostly radial polarization, and its structure is different from that shown in Fig. 3(b)-(c).

Other nonlinear simulations performed for lower values of the kinetic parameter $\bar{s}$, show the growth of both the $n=2$ and $n=3$ modes after the saturation of the tilt instability in the nonlinear regime. In the simulations with $E=4$ and $\bar{s}=1.6$, for example, the amplitudes of the $n=2$ and $n=3$ modes are comparable in the nonlinear phase. The simulation results for a case with $E=6.25$ and $\bar{s}=1.2$ are shown in Fig. 8, where the contours of axial magnetic field in the FRC midplane, and the surface plot of the plasma density are shown at $t=68 t_{A}$. The linear analysis for this equilibrium (assuming zero ion rotation) has shown that both the $n=2$ and $n=3$ modes are completely stabilized by FLR effects [Fig.3(a)]. However, the strong $n=3$ distortion of the plasma profiles is clearly seen in Fig. 8. The growth of the $n=3$ mode correlates with the significant ion toroidal rotation, which for this configuration is of order $V_{\phi, i} \sim 0.2 V_{A}$ for $t>40 t_{A}$. Note that the ion rotation frequency in this case is larger than the $n=1$ tilt instability growth rate $\Omega_{i} \gtrsim(3-4) \gamma$, and it is likely to have a strong stabilizing effect on the tilt mode.

The nonlinear behavior of the $n>1$ modes is generally similar in different simulation runs, but the detailed nonlinear evolution of these modes is sensitive to the choice of system parameters, including the resistivity profile, the initial perturbation amplitude, and even the numerical resolution. This sensitivity is due to the time evolution of the background plasma, including the magnetic field decay and the ion spin-up, and perhaps due to the dependence of the details of the ion toroidal spin-up on the simulation scheme. We have also found that simulations with higher numerical noise level (i.e., a smaller number of simulation particles), usually result in lower satu- 
ration amplitudes of the unstable modes.

\section{NONLINEAR SIMULATIONS AT HIGHER $\overline{\boldsymbol{s}}$}

Hybrid simulations in the small gyroradius regime, i.e., with values of $\bar{s}$ considerably larger than those in Section IV (i.e. $\bar{s} \gtrsim 7$ ), show MHD-like linear growth of the tilt mode and no nonlinear saturation. However, the nonlinear evolution of the instability in these runs has been found to be slower than that in similar MHD simulations. To make certain that the periodic boundary conditions used in our runs have a negligible effect on the observed nonlinear behavior, we have modified the numerical scheme to allow particle losses at the simulation boundaries. Two cases have been considered, one without a mirror field at the FRC ends, and the other with a mirror field (mirror ratio 1.5). The PIC scheme was used instead of the delta-f method to allow for particle losses. The simulations have been performed for a configuration with $E=4$ and $\bar{s}=7.4$ ( $\left.S^{*} \approx 60\right)$. The linear growth rate of the $n=1$ tilt mode in this case is close to that of the MHD model with $\gamma \gtrsim 0.9 \gamma_{m h d}$.

Simulations performed without the mirror field show a significant loss of particles (about 30 $\%$ ) during the linear phase of the instability. It is found that particle loss along the open field lines has a destabilizing effect on the $n=1$ tilt mode, increasing the linear growth rate by $\approx 10 \%$, probably due to a reduction of plasma pressure on the open field lines. In these simulations the nonlinear evolution of the instability is significantly slower than that in the MHD simulations. Thus, despite the loss of about one-half of the particles, the field reversal of $B_{z} \approx-0.5 B_{\text {ext }}$ is still present by $t=32 t_{A}$. In contrast, the configuration is destroyed in $\sim 15 t_{A}$ in the MHD simulations with the same equilibrium parameters. Significant ion spin up in the toroidal (diamagnetic) direction is also observed at $t>20 t_{A}$, with $V_{\phi, i} \approx 0.3 V_{A}$. 
Simulations performed for the same plasma parameters, but including the end mirror coils are shown in Figs. 9 and 10. The energy plots for the $n=0-4$ modes and the change in the total number of particles versus time are plotted in Fig. 9. These simulations show improved particle confinement, with a total particle loss of about $25 \%$ by the end of the run. The ion toroidal spin-up is smaller $\left(V_{\phi, i} \sim 0.1 V_{A}\right)$ than in the no-mirror-field case, and almost no reversed field remains at $t=30 t_{A}\left(B_{z} \lesssim-0.1 B_{e x t}\right)$.

In the high- $\bar{s}$ simulations, the $n=1$ mode is the dominant mode through the linear and nonlinear phases of the instability. The $n=1$ mode shows no nonlinear saturation, and the amplitudes of the $n \geq 2$ modes remain small. The growth of the $n=0$ component of the ion kinetic energy in Fig. 9(a) corresponds to the ion toroidal spin-up and also the axial motion, which breaks the plasma into two parts after it tilts over at $t \approx 15 t_{A}$. This can be seen in Fig. 10, where contours of the plasma density in the poloidal plane are shown at different times. The slow (compared to the MHD) nonlinear evolution of the tilt mode in these hybrid simulations can be related to the reduction of the kinetic parameter $\bar{s}$ in the nonlinear phase of instability (due the partial loss of poloidal flux), and the ion toroidal spin-up. Both of these effects are absent in the MHD model.

\section{CONCLUSIONS}

The results of hybrid simulations presented in this paper offer a definitive explanation of the stability properties observed in low $-\bar{s}$ FRC experiments. It has been demonstrated that, although the $n=1$ tilt mode is linearly the most unstable mode for nearly all experimentally-relevant FRC equilibria, it saturates nonlinearly without destroying the configuration, provided the FRC kinetic parameter, $\bar{s}$, is sufficiently small. The saturation of the $n=1$ tilt instability occurs in the presence 
of ion toroidal rotation, and is accompanied by the growth of the $n \geq 2$ rotational instabilities, which are often seen in experiments.

The saturation of the $n=1$ tilt instability, discovered in the low- $\bar{s}$ simulations, occurs due to the nonlinear change in the ion distribution function, and the stabilizing effects of ion sheared rotation and the nonlinear interaction with growing $n \geq 2$ rotational modes. The unstable equilibrium evolves nonlinearly into a new (rotating) configuration with smaller $\bar{s}$, larger $E$, and an increased separatrix beta.

The properties of the rotational modes, observed in the nonlinear phase of the tilt instability, are similar to those seen in FRC experiments, and they are in general agreement with previous theoretical studies of rotational instabilities ${ }^{18}$. The growing $n=2$ and $n=3$ modes have the lowest possible $k_{\|}$, and values of the rotational parameter $\alpha$ are of order $0.5-1$. The rotational instabilities are found to be stronger in the smaller- $\bar{s}$, more kinetic configurations. The details of the ion toroidal spin-up determine the nonlinear evolution of these instabilities.

In summary, the hybrid simulations presented here show that, while ion FLR effects determine the linear stability properties of non-rotating FRCs, the inclusion of nonlinear and ion toroidal flow effects is necessary for a satisfactory description of plasma behavior in low- $\bar{s}$ FRC experiments. High- $\bar{s}$ simulations show MHD-like linear growth of the tilt mode and no nonlinear saturation. The nonlinear evolution of the instability in these runs has been found to be slower than that in similar MHD simulations due to the nonlinear reduction of the kinetic parameter $\bar{s}$ and the ion toroidal spin-up. It is also found that the particle loss along open field lines has a destabilizing effect on the tilt mode, and contributes to the ion spin up in the toroidal direction. Additional means of stabilization (possibly shaping, or neutral beam injection) are required to achieve stability in reactor-scale, large- $\bar{s}$ FRCs. 


\section{ACKNOWLEDGMENTS}

This research was supported by the U.S. Department of Energy under Contract No. DEAC02-76CH03073. 


\section{APPENDIX A: Modified nonlinear delta-f scheme}

When the marker distribution is chosen to be the same as the physical ion distribution function, i.e., $g=f$, the particle weight can be expressed as

$$
w=\delta f / f=1-f_{0} / f=1-\exp \left[\left(\varepsilon_{0}-\varepsilon(t)\right) / T_{0}\right]
$$

where we have made use of $f(t)=f_{0}\left(t_{0}\right)=A \exp \left(-\varepsilon_{0} / T_{0}\right)$ and $f_{0}(t)=A \exp \left(-\varepsilon / T_{0}\right)$, where $\varepsilon_{0}$ is the initial ion energy. It can be seen from Eq. (A1), that the particle weight can grow to large (negative) values in the regions of phase space where $f$ is small compared to $f_{0}$ (or equivalently, when the particle energy is reduced compared to its initial value). It follows from Eq. (A1) that values of $w$ are restricted by $1-\exp \left(\varepsilon_{0} / T_{0}\right) \leq w<1$.

For particles from the tails of the Maxwellian distribution, the absolute value of the weight can grow to $\sim 10^{4}$, provided the ion distribution function is significantly modified compared to its initial form, and assuming that the cutoff velocity is $v_{\max }=3 v_{t h}$. The growth of the particle weights corresponds to sampling of $f_{0}$ with a small (insufficient) number of marker particles in the regions of phase space where $f$ becomes very small. In the simulations, this effects can be seen at relatively large amplitudes, when a small fraction of particles (say less than $0.1 \%$ ) has large (negative) weight, which is several orders-of-magnitude larger than the average weight. This results in a loss of accuracy, and can be manifest in the violation of numerical conservation properties, including the conservation of the number of particles $\left(\delta N=\sum_{m} w_{m}=0\right)$ and the total energy.

Since at large amplitudes the separation of the distribution function into known (equilibrium) and perturbed parts does not provide an advantage, a numerical scheme that permits a switch to the conventional PIC simulation method has been implemented. The hybrid (delta-f-PIC) scheme can be expressed as a weighted average of delta-f and PIC methods 


$$
n=(1-\lambda) n_{0}+\sum_{m}\left[(1-\lambda) w_{m}+\lambda p_{m}\right] S\left(\mathbf{x}-\mathbf{x}_{m}\right)
$$

where $\lambda$ is a parameter (dependent on time or the perturbation amplitude), which allows us to switch from the delta-f $(\lambda=0)$ to PIC $(\lambda=1)$ description, and $p=f / g$ is an equilibrium (constant) weight in the PIC simulations. A similar modification applies to the current density calculation in Eq. (10). Note that Eq. (A2) does not require significant additional computations. It provides a low-noise scheme at small amplitudes, and regular PIC accuracy and numerical noise level at large amplitudes. The accuracy of the results can be analyzed by direct comparison with PIC simulations for a test case.

An alternate approach to mitigate the accuracy loss of the delta-f scheme at large amplitudes can be the choice of $g=$ const, so that the marker particles are loaded uniformly in phase space, and $w \sim \delta f$ is always limited. In this case, however, the entire phase space needs to be sampled, which results in a higher numerical noise level and poor resolution, unless the number of simulation particles is significantly increased. The uniform sampling also produces large variations in particle equilibrium weights $p_{m} \sim \exp \left(-\varepsilon_{0} / T_{0}\right)$. A somewhat better solution may be the use of a padded equilibrium distribution function for numerical loading, i.e., $g=f+C$, where $C$ is a small constant, so that $|w|$ is limited by $\sim 1 / C$, and the regions of phase space where $f_{0}$ is finite are always well sampled.

In the HYM code, the scheme given by Eq. (A2) has been implemented, and the convergence of nonlinear results is validated by varying the form of the $\lambda$ dependence on the perturbation amplitude and increasing the number of simulation particles. 
${ }^{1}$ M. Tuszewski, Nucl. Fusion 28, 2033 (1988).

${ }^{2}$ S. Ohi, T. Minato, Y. Kawakami et al., Phys. Rev. Lett. 51, 1042 (1983); A. L. Hoffman, J. T. Slough, D. G. Harding, Phys. Fluids 26, 1626 (1983); R. E. Siemon, W. T. Armstrong, D. C. Barnes et al., Fusion Technol. 9, 13 (1986).

${ }^{3}$ J. T. Slough and A. L. Hoffman, Phys. Fluids 5, 4366 (1993).

${ }^{4}$ A. L. Hoffman, L. N. Carey, E. A. Crawford et al., Fusion Technology 23, 185 (1993).

${ }^{5}$ M. Tuszewski, D. C. Barnes, R. E. Chrien, J. W. Cobb, D. J. Rej, R. E. Siemon, D. P. Taggart, B. L. Wright, Phys. Rev. Lett. 66, 711 (1991); M. Tuszewski et al., Phys. Fluids B 3, 2856 (1991).

${ }^{6}$ R. D. Milroy, D. C. Barnes, R. C. Bishop, R. B. Webster, Phys. Fluids B 1, 1225 (1989).

${ }^{7}$ A. Ishida, H. Momota and L. C. Steinhauer, Phys. Fluids 31, 3024 (1988).

${ }^{8}$ L. C. Steinhauer and A. Ishida, Phys. Fluids B 2, 2422 (1990).

${ }^{9}$ D. C. Barnes, Phys. Plasmas 9, 560 (2002); 8, 4856 (2001).

${ }^{10}$ H. Yamada, T. Katano, A. Ishida, L. C. Steinhauer, Phys. Plasmas 10, 1168 (2003).

11 Y. A. Omelchenko, M. J. Schaffer, and P. B. Parks, Phys. Plasmas 8, 4463 (2001).

${ }^{12}$ H. Ohtani, R. Horiuchi, and T. Sato, Phys. Plasmas 10, 145 (2003).

${ }^{13}$ E. V. Belova, R. C. Davidson, H. Ji, M. Yamada, Phys. Plasmas 10, 2361 (2003).

${ }^{14}$ E.V. Belova, S. C. Jardin, H. Ji, M. Yamada, R. M. Kulsrud, Phys. Plasmas 7, 4996 (2000). 
${ }^{15}$ N. Iwasawa, A. Ishida and L. C. Steinhauer, Phys. Plasmas 8, 1240 (2001).

${ }^{16}$ E. V. Belova, S. C. Jardin, H. Ji, R. M. Kulsrud, W. Park, and M. Yamada, 18th IAEA Fusion Energy Conference, Sorrento, 2000 (International Atomic Energy Agency, Vienna, 2000), paper IAEA-CN77/THP2/02.

${ }^{17}$ S. E. Parker and W. W. Lee, Phys. Fluids 5, 77 (1993).

${ }^{18}$ D. S. Harned, Phys. Fluids 26, 1320 (1983); C. E. Seyler, 22, 2324 (1979) 


\section{FIGURE CAPTIONS}

FIG.1. Normalized growth rates obtained in linear hybrid simulations of the $n=1$ tilt mode instability for elliptic FRC equilibria with E=6.25 and $P_{e}=0$ (thin), and finite $P_{e}$ (thick). The dashed line shows the empirical scaling in Eq. (11).

FIG.2. (a) Growth rates of the $n=1, n=2$, and $n=3$ modes plotted versus the $1 / \bar{s}$ parameter. Linear structure of $n=2$ mode; (b) contour plots of the axial velocity at the midplane cross section; and (c) vector plot of the poloidal velocity in the $R-z$ plane.

FIG.3. (a) Radial profiles of the ion toroidal flow velocity at the FRC midplane at $t=46 t_{A}$ and $t=80 t_{A}$; and (b) contour plot of $V_{\phi}$ showing one-quarter of the cylinder $r-z$ plane.

FIG.4. Time evolution of the $n=0-4$ Fourier harmonics of the ion kinetic energy obtained from hybrid simulations with $E=6.25$ and $\bar{s}=2.4$.

FIG.5. Contour plots of ion density in the poloidal $r-z$ plane obtained from the simulations shown in Fig. 4. Time is normalized to the Alfvén time.

FIG.6 Vector plot of the $n=0$ component of the poloidal magnetic field at $t=80 t_{A}$ obtained from the simulations shown in Fig. 4.

FIG.7 (a) Time evolution of the $n=0-4$ Fourier harmonics of the ion density perturbation; and (b) contour plots of $\delta n$ at the toroidal cross section at $t=60 t_{A}$ and $t=76 t_{A}$.

FIG.8 (a) Contours of the axial magnetic field at the FRC midplane; and (b) surface plot of the ion density at $t=68 t_{A}$ obtained from nonlinear simulations with $E=6.25$ and $\bar{s}=1.2$.

FIG.9. (a) Time evolution of the $n=0-4$ Fourier harmonics of the ion kinetic energy obtained from hybrid simulations with $E=4$ and $\bar{s}=7.4$; and (b) time evolution of the total number of ions in the configuration.

FIG.10. Contour plots of the ion density in the poloidal $r-z$ plane obtained from the simu- 
lations shown in Fig. 9. Time is normalized to the Alfvén time. 


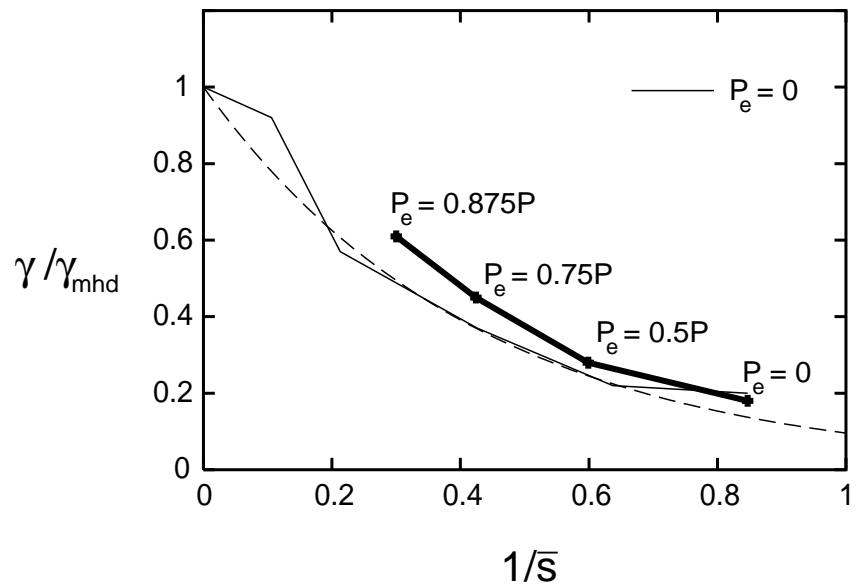

Figure 1.

Belova, Physics of Plasmas 

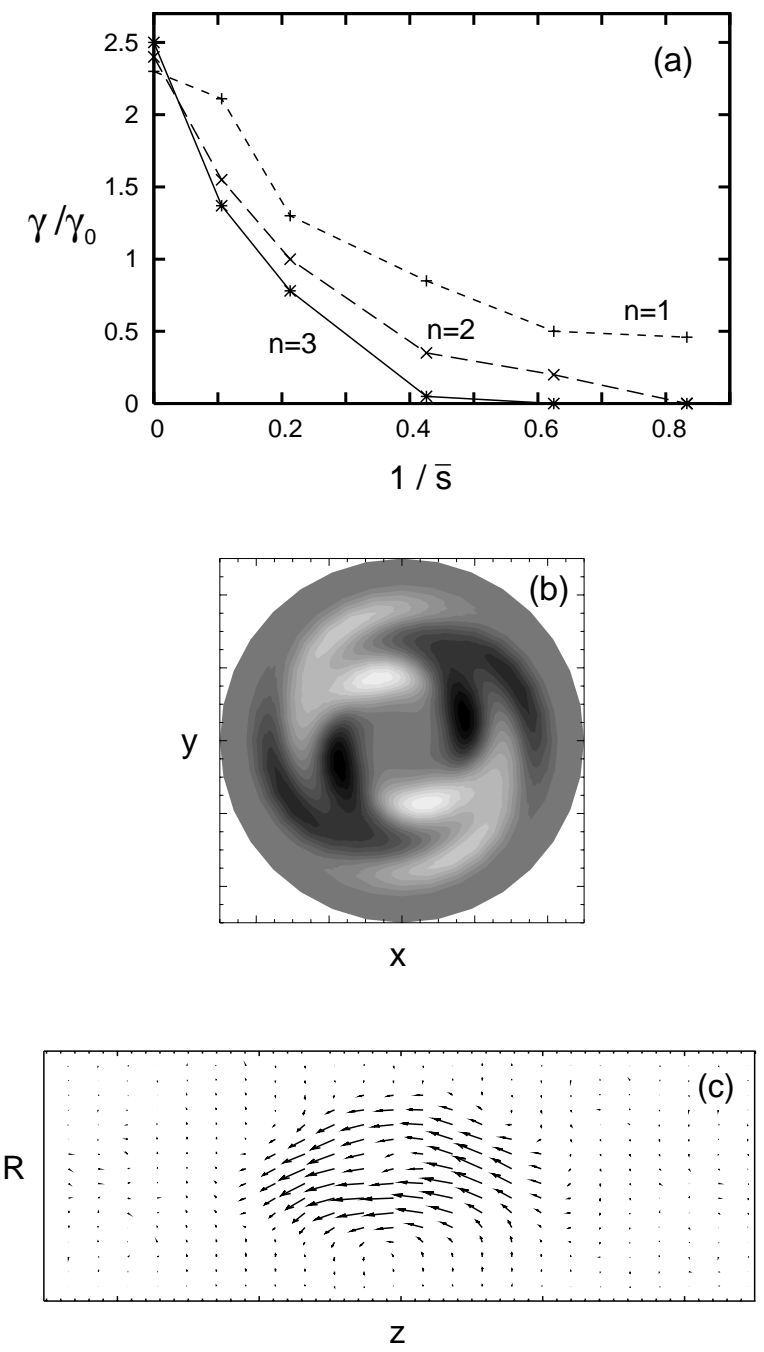

Figure 2.

Belova, Physics of Plasmas 

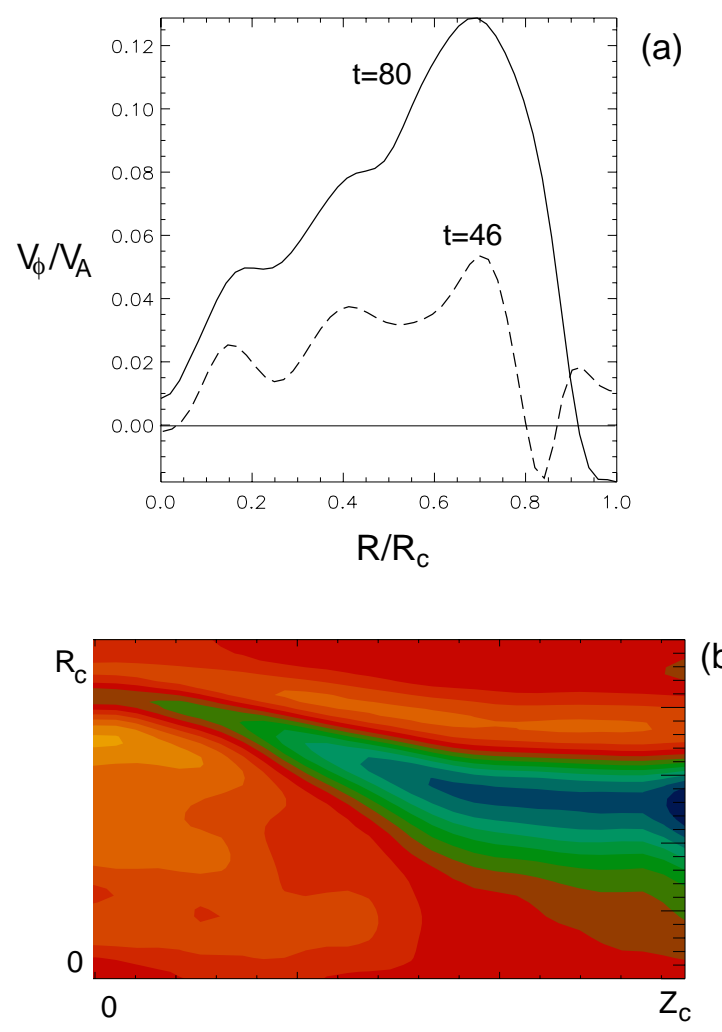

Figure 3.

Belova, Physics of Plasmas 


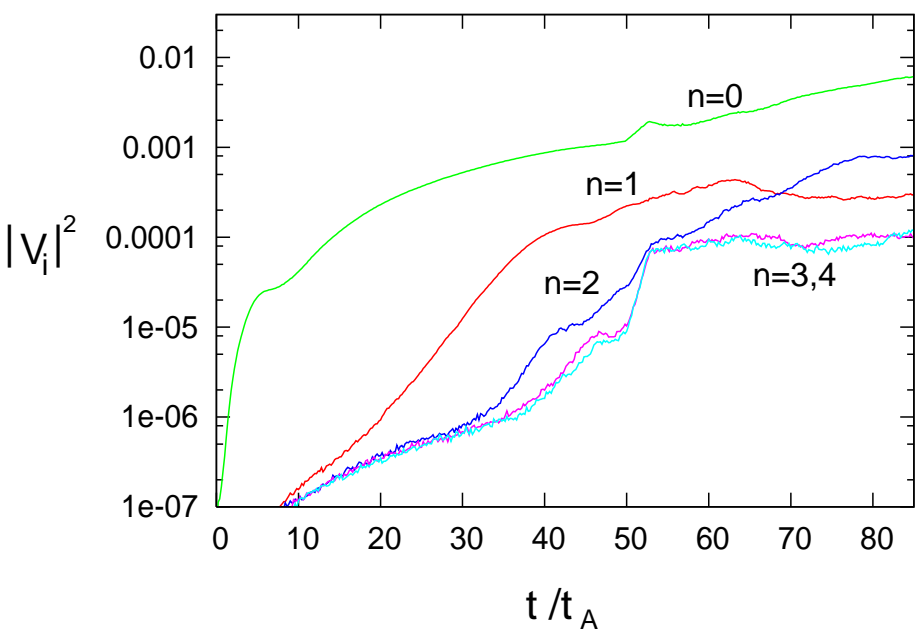

Figure 4.

Belova, Physics of Plasmas 


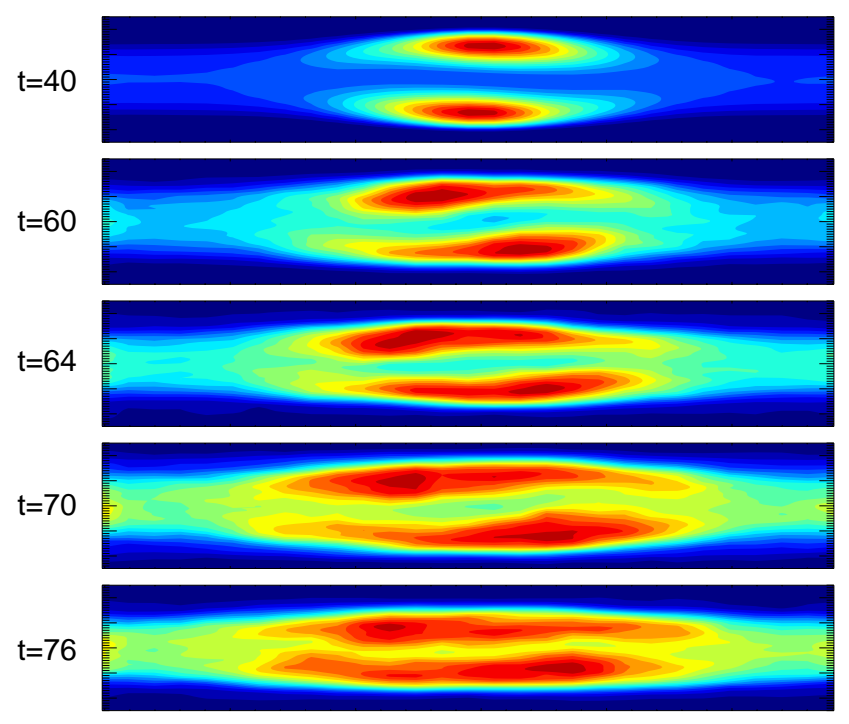

Figure 5.

Belova, Physics of Plasmas 


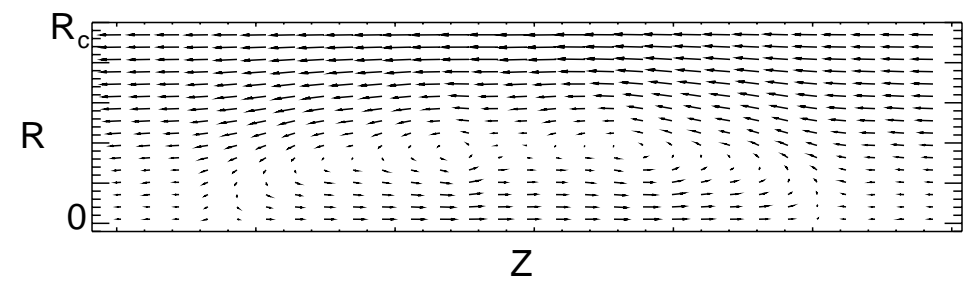

Figure 6.

Belova, Physics of Plasmas 


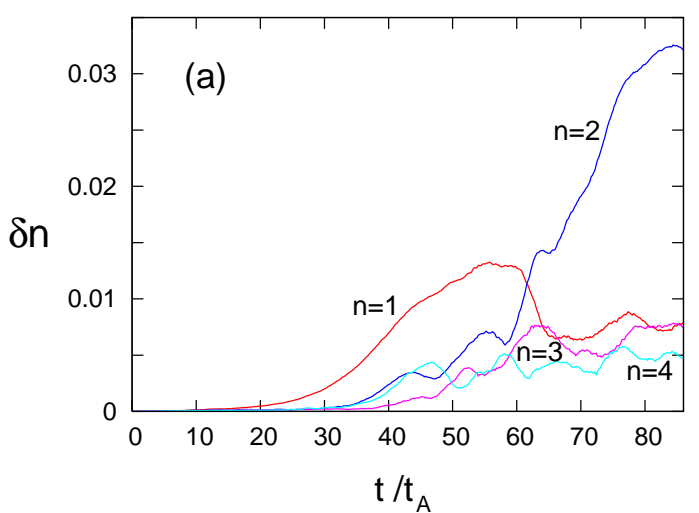

(b)

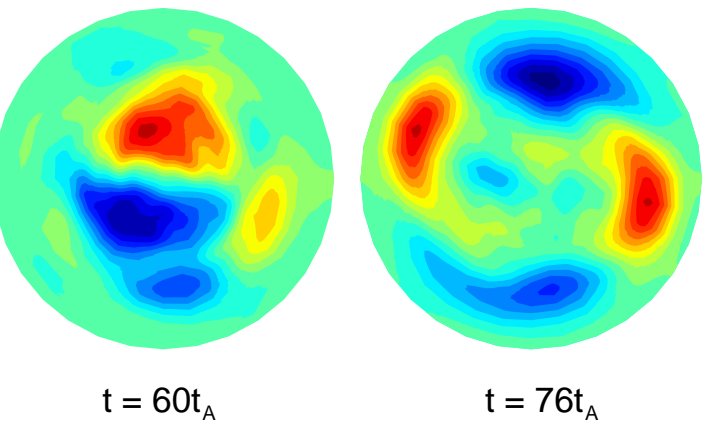

Figure 7.

Belova, Physics of Plasmas 


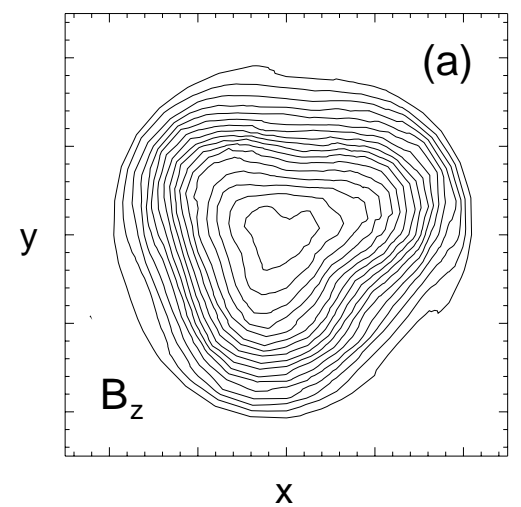

(b)

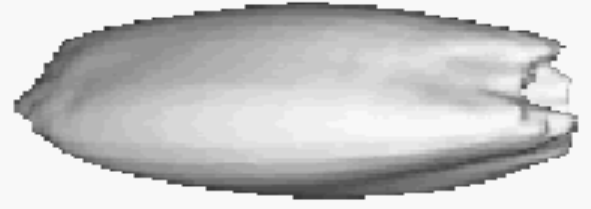

Figure 8.

Belova, Physics of Plasmas 


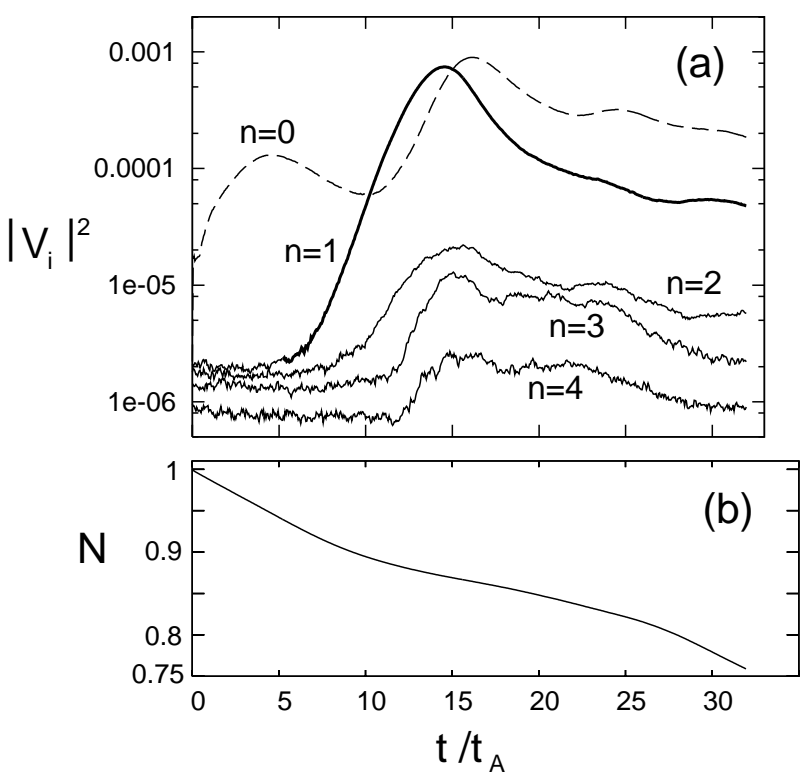

Figure 9.

Belova, Physics of Plasmas 


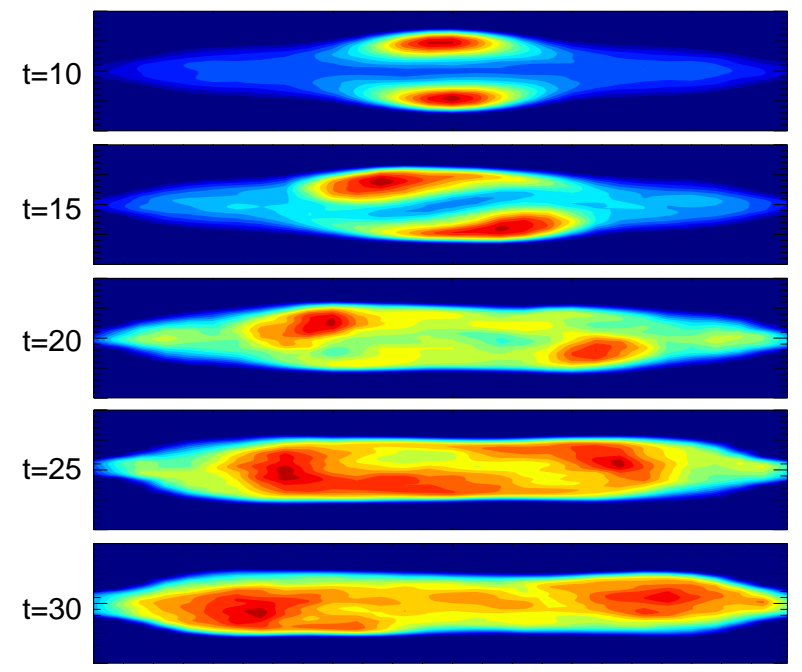

Figure 10.

Belova, Physics of Plasmas 


\section{External Distribution}

Plasma Research Laboratory, Australian National University, Australia

Professor I.R. Jones, Flinders University, Australia

Professor João Canalle, Instituto de Fisica DEQ/IF - UERJ, Brazil

Mr. Gerson O. Ludwig, Instituto Nacional de Pesquisas, Brazil

Dr. P.H. Sakanaka, Instituto Fisica, Brazil

The Librarian, Culham Laboratory, England

Mrs. S.A. Hutchinson, JET Library, England

Professor M.N. Bussac, Ecole Polytechnique, France

Librarian, Max-Planck-Institut für Plasmaphysik, Germany

Jolan Moldvai, Reports Library, Hungarian Academy of Sciences, Central Research Institute for Physics, Hungary

Dr. P. Kaw, Institute for Plasma Research, India

Ms. P.J. Pathak, Librarian, Institute for Plasma Research, India

Ms. Clelia De Palo, Associazione EURATOM-ENEA, Italy

Dr. G. Grosso, Instituto di Fisica del Plasma, Italy

Librarian, Naka Fusion Research Establishment, JAERI, Japan

Library, Laboratory for Complex Energy Processes, Institute for Advanced Study, Kyoto University, Japan

Research Information Center, National Institute for Fusion Science, Japan

Dr. O. Mitarai, Kyushu Tokai University, Japan

Dr. Jiangang Li, Institute of Plasma Physics, Chinese Academy of Sciences, People's Republic of China

Professor Yuping Huo, School of Physical Science and Technology, People's Republic of China

Library, Academia Sinica, Institute of Plasma Physics, People's Republic of China

Librarian, Institute of Physics, Chinese Academy of Sciences, People's Republic of China

Dr. S. Mirnov, TRINITI, Troitsk, Russian Federation, Russia

Dr. V.S. Strelkov, Kurchatov Institute, Russian Federation, Russia

Professor Peter Lukac, Katedra Fyziky Plazmy MFF UK, Mlynska dolina F-2, Komenskeho Univerzita, SK-842 15 Bratislava, Slovakia

Dr. G.S. Lee, Korea Basic Science Institute, South Korea

Institute for Plasma Research, University of Maryland, USA

Librarian, Fusion Energy Division, Oak Ridge National Laboratory, USA

Librarian, Institute of Fusion Studies, University of Texas, USA

Librarian, Magnetic Fusion Program, Lawrence Livermore National Laboratory, USA

Library, General Atomics, USA

Plasma Physics Group, Fusion Energy Research Program, University of California at San Diego, USA

Plasma Physics Library, Columbia University, USA

Alkesh Punjabi, Center for Fusion Research and Training, Hampton University, USA

Dr. W.M. Stacey, Fusion Research Center, Georgia Institute of Technology, USA

Dr. John Willis, U.S. Department of Energy, Office of Fusion Energy Sciences, USA

Mr. Paul H. Wright, Indianapolis, Indiana, USA 
The Princeton Plasma Physics Laboratory is operated by Princeton University under contract with the U.S. Department of Energy.

\author{
Information Services \\ Princeton Plasma Physics Laboratory \\ P.O. Box 451 \\ Princeton, NJ 08543
}

Phone: 609-243-2750

Fax: 609-243-2751

e-mail: pppl_info@pppl.gov

Internet Address: http://www.pppl.gov 